\title{
The external rotation method for reduction of acute anterior shoulder dislocations
}

\author{
Mario Marinelli · Luigi de Palma
}

Received: 6 January 2008 / Accepted: 17 November 2008/Published online: 8 January 2009

(c) Springer-Verlag 2008

\begin{abstract}
Background Shoulder dislocations account for almost $50 \%$ of all joint dislocations, and are most commonly anterior (90-98\%) and occur due to trauma. This prospective study was conducted to report our experiences of using the external rotation method (ERM) in the reduction of acute anterior shoulder dislocation.

Materials and methods Between August 2006 and April 2007, ERM was applied to 31 patients who presented with traumatic anterior shoulder dislocation to the Emergency Department of our Hospital which is a level 2 trauma centre. We evaluated the type of dislocation, the effectiveness of the procedure in achieving reduction, the need for premedication, the ease of performing the reduction, and complications, if any. Results Of the 31 patients, 29 had a successful reduction. No premedication was required in 25 patients who had a successful reduction, and the average time required for reduction in 25 was less than 2 min. Only five patients reported severe pain during the process of reduction. The method was not successful in two patients in whom the reduction was achieved under narcosis. We experienced a success rate of $89 \%$ at the first attempt. None of the patients encountered any complication.

Conclusions ERM for the reduction of acute anterior dislocation of the shoulder is a safe and reliable method, mainly without requirement for any sedatives or opiate analgesics, that can be performed relatively painlessly for anterior shoulder dislocations. As no single method has a $100 \%$ success rate, ERM is a useful one to know.
\end{abstract}

M. Marinelli · L. de Palma ( $\square)$

Cattedra di Ortopedia e Traumatologia, Università Politecnica delle Marche, Azienda Ospedaliero-Universitaria, Ospedali Riuniti di Ancona, Via Conca, Torrette, 60100 Ancona, Italy e-mail: 1.depalma@univpm.it
Keywords Acute shoulder dislocation . Extra rotation method

\section{Introduction}

Shoulder dislocations account for almost $50 \%$ of all joint dislocations presenting to emergency departments [1]. Very often, shoulder dislocations are anterior (90-98\%) and occur as a result of trauma [2, 3]. Ideal reduction methods should be quick, effective, and as painless as possible for patients; and they should not cause iatrogenic complication. Traditional techniques to reduce the dislocated glenohumeral joint can be painful to the patient and may also be associated with further injury [4-6]. The external rotation method is a relatively new technique, which is reported to be safe, comfortable, and reliable [7]. This prospective study was conducted to report our experiences of using the external rotation method (ERM) to reduce traumatic anterior shoulder dislocations and to evaluate the causes of failure.

\section{Materials and methods}

A prospective study was conducted in the Emergency Department of the Azienda Ospedaliero-Universitaria Ospedali Riuniti of Ancona. Between August 2006 and April 2007, 31 patients with traumatic anterior shoulder dislocation presented to our hospital. ERM was applied to these patients by one full-time orthopaedic surgeons. Written informed consent was obtained from each patient. The cohort of consecutive patients with acute anterior dislocation of the shoulder who were initially treated with 
use of the ERM consisted of 12 women (38.7\%) and 19 men $(61.3 \%)$, mean age 45 years (range $17-55$ years). There were no shoulders with general hyperlaxis. Right anterior shoulder dislocation was diagnosed in 22 (70.9\%). Fourteen patients $(45.1 \%)$ had a history of recurrent shoulder dislocations. The mechanisms of injury were simple fall and contact sports. The dislocation was subcoracoid for 23 patients and subglenoid for 8 patients. Patients presented to our emergency department a mean of $1 \mathrm{~h}$ after trauma. One patient presented $2 \mathrm{~h}$ after the dislocation.

Demographic data, dislocation site, fracture of the greater tuberosity and/or a Hill-Sachs compression defect existence, dislocation history (for example, recurrent dislocation), and manipulation attempts were recorded on data sheets. Exclusion criteria included polytrauma, and dislocations associated with Neer proximal humeral fractures or with glenoid fractures. Conventional antero-posterior (AP) and transthoracic view radiography were performed to identify anterior shoulder dislocation after all patients had undergone a brief neurovascular examination.

The study was performed in accordance with the ethical standards of the 1964 Declaration of Helsinki and was approved by the Ethical Committee of our Institution.

\section{External rotation technique}

With the patient in the supine position the initial manoeuvre is traction on the arm as it is held at $90^{\circ}$ elbow flexion, then the arm is adducted to the side of the chest and the shoulder is placed in $20^{\circ}$ forward flexion. The orthopaedic surgeon grasps the wrist and uses it as a guide, then the shoulder is externally rotated until the forearm is in the coronal plane (Fig. $1 \mathrm{a}, \mathrm{b}, \mathrm{c}, \mathrm{d}$ ). Once reduction is achieved, the arm is internally rotated to bring the forearm into the abduction position and is immobilized using a sling (IR-15 ${ }^{\circ}$ ABD with a pillow). Discharge decisions were given after neurovascular examinations and post-reduction control radiographic evaluation.

Sedation was used as and when needed after more of $15 \mathrm{~min}$ of vain attempts $(0.05 \mathrm{mg} / \mathrm{kg}$ midazolam through an intravenous cannula). Complications, such as injury to the axillary nerve, vascular compromise, and iatrogenic fracture attributable to the reduction method, were also noted.

\section{Results}

The patients were treated in the orthopaedic room at time zero by one of us, after which diagnosis was confirmed by clinical examination and radiographic evaluation with use of antero-posterior and axillary radiographs. The patient was informed briefly about the method of reduction while lying in the supine position. Reduction of the shoulder dislocation was achieved within 5 min in 20 patients $(64.5 \%)$ and within $10 \mathrm{~min}$ in eight patients $(25.8 \%)$. The average time to reduction was $3 \mathrm{~min}[95 \%$ confidence interval (CI) $1.5-8.5 \mathrm{~min}]$. No sedative or any opiate analgesic was needed for 25 patients $(80.6 \%)$. None of the patients encountered any complication (including the patients requiring sedation).

None of them had concomitant fractures and in 18 patients a Hill-Sachs lesion was recorded. We experienced a success rate of $89 \%$ after the first attempt. The method was unsuccessful in two patients with subglenoid dislocation. Neither of them had previously dislocated the shoulder. The dislocations were subsequently reduced, with the patient under narcosis, with use of the traditional method.

Of the six patients treated with premedication, three had a successful reduction and reported mild or moderate pain. Of the 25 patients treated without premedication who had a successful reduction six patients reported severe pain.

All patients with a history of recurrent dislocation of the shoulder $(45.1 \%)$ had a successful reduction with use of ERM and reported that the reduction was more easily done with this method than with the previous methods. The mean duration of hospitalization in Emergency Department for the 29 patients treated successfully with the ERM was 55 mins (range 40-95 mins).

\section{Discussion}

Recently, Kuhn [8] presented the best available evidence to answer questions regarding the treatment of patients with an initial anterior shoulder dislocation, reporting that, of the many methods to reduce the dislocated shoulder, little data exist to identify the best method and that premedication with intra-articular lidocaine has fewer complications and requires a shorter time in the emergency room than intravenous sedation, with no detectable differences in reduction success rates. Ashton and Hassan [9] carried out a review to establish whether Kocher's or Milch's technique was better at reducing shoulder dislocations. The clinical bottom line was that individual physician preference for either Kocher's or Milch's method of reduction is not supported by the evidence.

In the literature numerous techniques for treating anterior shoulder dislocation have been reported, such as traction-countertraction, and the Milch [10] and modified Stimson [11] manoeuvres. These techniques use traction, and/or leverage of the humerus, often requiring considerable force and causing significant patient discomfort: traction increases muscle spasm and may make reduction 
Fig. 1 The external rotation method for the reduction of an acute anterior dislocation of the shoulder. a The patient is in the supine position with the elbow in $90^{\circ}$ flexion. b The arm is adducted to the side of the chest and the shoulder is placed in $20^{\circ}$ forward flexion. $\mathbf{c}$ The shoulder is externally rotated until the forearm is in the coronal plane. d The arm is internally rotated to bring the forearm into the abduction position
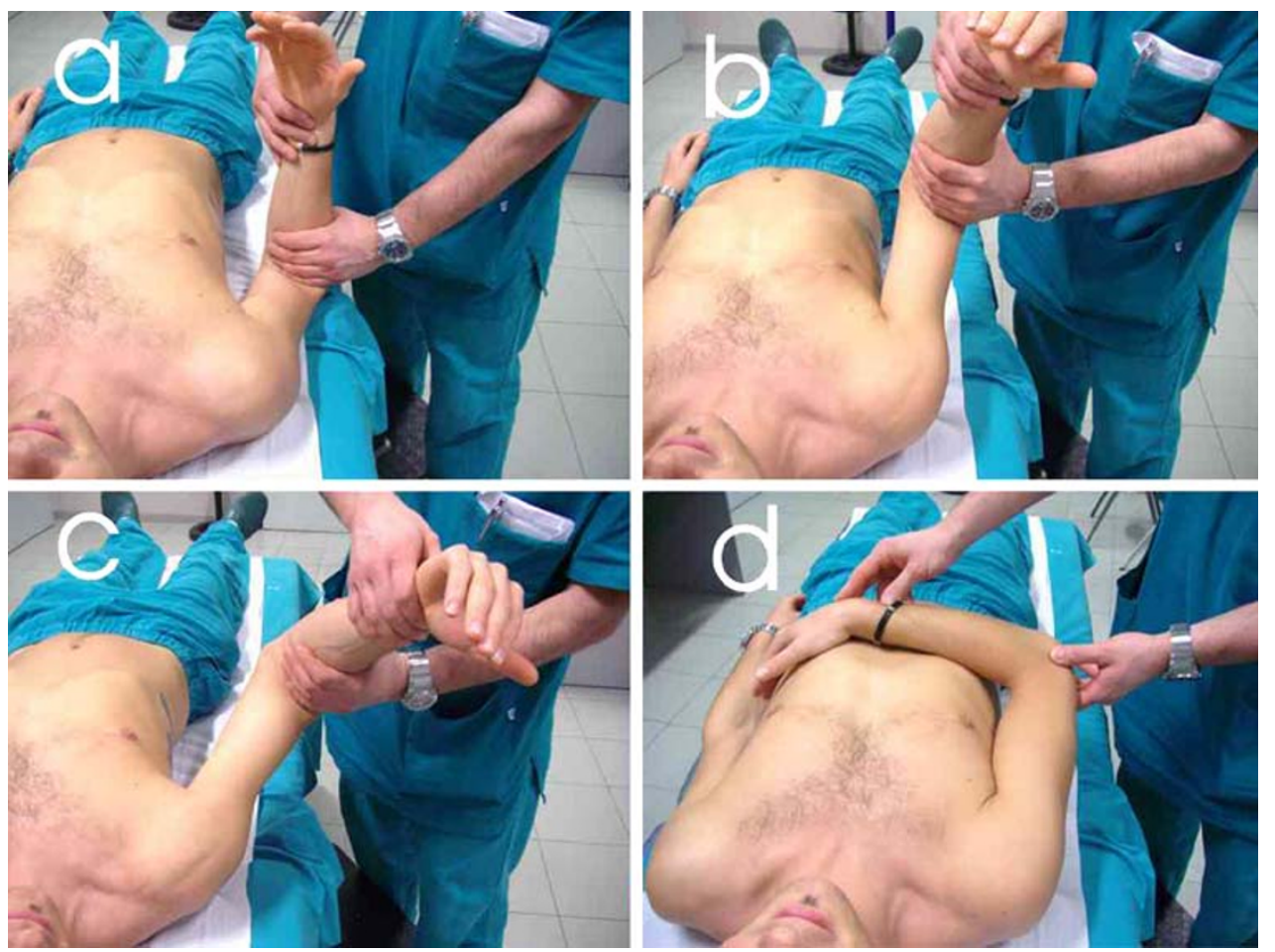

difficult, more painful, and less likely to succeed $[12,13]$. More traditional techniques (Hippocratic method and the Kocher manoeuvre) are no longer recommended because of a high incidence of associated complications, such as axillary nerve injury, humeral shaft and neck fractures, and capsular damage [14].

Because ideal reduction methods should be quick and effective, painless reduction of acute anterior shoulder dislocations without anesthesia gained popularity.

Yuen et al. [15], in support of an easy method for reducing anterior shoulder dislocation by junior emergency medicine residents, reported an overall success rate of $87.5 \%$ for the Spaso technique, which is simple, effective, and able to be performed by single operator.

Baykal [16] and colleagues reported the experiences of using the scapular manipulation technique to reduce traumatic anterior shoulder dislocations. They reported a success rate of $90.2 \%$ at first attempt, mainly without requirement for any sedatives or opiate analgesics.

The Milch technique consists of shoulder abduction and external rotation with "pulsion" of the humeral head. O'Connor [17] studied the effectiveness of this technique on 76 consecutive acute anterior shoulder dislocations in 75 patients. All 76 shoulders were reduced on first attempt. No anesthesia was used, and no complications were reported from the reduction manoeuvre.

Our study demonstrated that ERM is easy to perform as we were able to achieve a closed reduction of acute anterior dislocation in $89 \%$ of the patients, requiring only a single physician to perform it. Our success rate is similar to that reported by Mirick [12] and Eachempati [18].

We place the patient in the supine position with the arm adducted to the side and the elbow in $90^{\circ}$ flexion. To facilitate relaxation of the anterior capsule of the shoulder and to prevent any bow-stringing action of the long head of the biceps and the conjoint tendon, we position the shoulder in $20^{\circ}$ forward flexion [19]. Finally, external rotation was used to neutralize the medially directed contraction force of the subscapularis and the pectoralis major muscle [20].

Various methods of anesthesia are available for reduction. Pradhan et al. [21] suggested that use of intra-articular lignocaine for reduction of shoulder dislocation is safe and effective, especially when patients present early to Emergency Room. In patients presenting late (more than $5 \mathrm{~h}$ ) intravenous anesthesia should be primarily considered as the method for shoulder reduction.

However, in our series, in 25 patients who had a dislocation for the first time (80.6\%), reductions were performed without the use of sedation.

Two subglenoid dislocations could not be reduced successfully. They had severe muscle spasm and pain and patients were very apprehensive with poor compliance. Reduction was achieved with use of the traction-countertraction method under narcosis.

Ideally, physicians working in the emergency department should become familiar with a number of techniques for reducing anterior dislocations of the shoulder, as no 
single method has a $100 \%$ success rate. ERM for the reduction of an acute anterior dislocation of the shoulder is a safe and reliable method, mainly without requirement for any sedatives or opiate analgesics, that can be performed relatively painlessly for anterior shoulder dislocations.

Conflict of interest statement Authors deny any commercial associations they have that might give rise to a conflict of interest in connection with the submitted article.

\section{References}

1. Blake R, Hoffman J (1999) Emergency department evaluation and treatment of the shoulder and humerus. Emerg Med Clin North Am 17:859-876

2. Hill JA (1993) Epidemiologic perspective on shoulder injuries. Clin Sports Med 2:241-247

3. Westin CD, Gill EA, Noyes ME (1995) Anterior shoulder dislocation: a simple and rapid method for reduction. Am J Sports Med 23:369-372

4. Beattie TF, Steedman DJ, McGowan A, Robertson CE (1986) A comparison of the Milch and Kocher techniques for acute anterior dislocation of the shoulder. Injury 17:349-352

5. Janecki CJ, Shahcheragh GH (1982) The forward elevation maneuver for reduction of anterior dislocations of the shoulder. Clin Orthop 164:177-180

6. Manes HR (1980) A new method of shoulder reduction in the elderly. Clin Orthop 147:200-222

7. Plummer D, Clinton J (1989) The external rotation method for reduction of acute anterior shoulder dislocation. Emerg Med Clin North Am 7:165-175

8. Kuhn JE (2006) Treating the initial anterior shoulder dislocation-an evidence-based medicine approach. Sports Med Arthrosc 14(4):192-198
9. Ashton HR, Hassan Z (2006) Best evidence topic report. Kocher's or Milch's technique for reduction of anterior shoulder dislocations. Emerg Med J 23(7):570-571

10. Milch H (1938) Treatment of dislocation of the shoulder. Surgery 3:732-738

11. Stimson LA (1900) An easy method of reduction dislocation of the shoulder and hip. Med Record 57:356

12. Mirick MJ, Clinton JE, Ruiz E (1979) External rotation method of shoulder dislocation reduction. JACEP 8:528-531

13. Russell JA, Holmes EM III, Keller DJ, Vargas JH III (1981) Reduction of acute anterior shoulder dislocations using the Milch technique: a study of ski injuries. J Trauma 21(9):802-804

14. Daya M (2002) Shoulder. In: Marx JA, Hockberger RS, Walls RM (eds) Rosen's emergency medicine. concepts and clinical practice, 5th edn. Mosby Inc, Missouri, pp 576-606

15. Yuen MC, Yap PG, Chan YT, Tung WK (2001) An easy method to reduce anterior shoulder dislocation: the Spaso technique. Emerg Med J 18(5):370-372

16. Baykal B, Sener S, Turkan H (2005) Scapular manipulation technique for reduction of traumatic anterior shoulder dislocations: experiences of an academic emergency department. Emerg Med J 22(5):336-338

17. O'Connor DR, Schwarze D, Fragomen AT, Perdomo M (2006) Painless reduction of acute anterior shoulder dislocations without anesthesia. Orthopedics 29(6):528-532

18. Eachempati KK, Dua A, Malhotra R, Bhan S, Bera JR (2004) The external rotation method for reduction of acute anterior dislocations and fracture-dislocations of the shoulder. J Bone Joint Surg Am 86-A(11):2431-2434

19. Leidelmeyer R (1977) Reduced! a shoulder, subtly and painlessly. Emerg Med 9:233-234

20. Zahiri CA, Zahiri H, Tehrany F (1997) Anterior shoulder dislocation reduction technique-revisited. Orthopedics 20:515-521

21. Pradhan RL, Lakhey S, Pandey BK, Rijal KP (2006) Reduction of acute anterior shoulder dislocations: comparing intraarticular lignocaine with intravenous anesthesia. JNMA J Nepal Med Assoc 45(162):223-227 